\title{
Geometric Methods in Head Impact Response
}

\author{
GUY S. NUSHOLTZ
}

Bioscience Division, The University of Michigan,

Transportation Research Institute, Room 406, 2901 Baxter Road,

Ann Arbor, Michigan 48109

Received 31 July 1987; revised 2 March 1988

\begin{abstract}
Geometric method currently being applied to fundamental physics problems can also be used in experimental mechanics. The problem of biokinematics of impact response, in particular head impact response, can be addressed through the use of the moving frame of Cartan and associated geometric structures.
\end{abstract}

\section{INTRODUCTION}

Understanding the natural world in terms of its physics and mechanics begins with a long tradition of qualitative investigation culminating with Kepler and Galileo in the sixteenth century. Over a period of time, quantitative theory characterized by concomitant developments in mechanics, mathematics, and philosophy of science arose. These works are epitomized by Newton, Euler, Lagrange, Laplace, Hamilton, and Jacobi. Much of this work, developed in the sixteenth through the nineteenth centuries, used a relatively simple geometric view of the three-dimensional Euclidean world and emphasized the great diversity of analytic techniques that are necessary to solve the different equations of physics. Two developments in this century, however, have markedly increased the role of geometry in the realm of physics and mechanics. The first is the development of the theory of relativity by Einstein, according to which the Euclidean three-space is only a subset of, and an approximation of, actual four-space of the physical world. The second development, initiated by Cartan and which is only very recently being felt, is that geometric methods lead naturally to the development of certain analytic tools (e.g., Lie derivatives and exterior calculus) and to certain concepts (i.e., manifolds, fiber bundles, and vectors with derivatives) which have great power when applied to the analysis of physical problems. 
Modern geometric techniques have become more and more important in theoretical physics, where they have lead to greater simplicity in applied mathematics and to a more fundamental understanding of physics. This revolution has affected not only special and general relativity, but other fields where the geometry involved is not always that of a physical space (i.e., the space associated with the distance between two material bodies), but rather that of a more abstract space of variables. This occurs in such diverse fields as electromagnetics, thermodynamics, Hamiltonian theory, fluid dynamics, and elementary-particle physics.

Geometric methods of physics and mechanics can be very useful in understanding the experimental mechanics associated with impacts biokinematics. This paper gives a brief overview of some concepts in geometric methods and shows the impact response of the human head can be investigated through the use of some of the geometric methods. In particular, the impact response of the head can be investigated using the moving frame of Cartan.

\section{BACKGROUND}

The following sections give brief nonrigorous overview of some of the techniques used in geometric mechanics. For a more complete explanation, see References [1-7]. In addition, a brief explanation of the University of Michigan Transportation Institute (UMTRI) experimental techniques associated with body-segment impact response are also presented. They are illustrated in this paper by the problems of head impact response. For a more complete discussion of UMTRI techniques, see References [8-16].

\section{MANIFOLD}

Most physical problems involve some sort of continuous "smooth" space. This could be three-dimensional or four-dimensional space in which the motion of most material bodies, in terms of displacements, are calculated. Or, just as easily, it could be the phase space of classical or quantum mechanics, in which momentum plays the part of one of the coordinates. The thermodynamic equilibrium states, or some still more abstract space, might also come to mind. The thing common to all these spaces is that they are continuous. A manifold (i.e., a differential manifold) is a continuous space with enough structure so that calculus may be applied. This, in turn, implies that given any point on the manifold, there can be found a neighborhood small enough (i.e., a local area) to behave as if it were Euclidean space of the same dimensions. Examples of manifolds are the curve traced by a point mass as it moves in space; the space of energy, temperature, entropy, pressure, and volume of a thermodynamic system; and the surface of a sphere or ellipsoid. 
CHART

It is convenient to have set coordinates to describe the manifold of interest. However, it is not always possible to find an origin and a set of coordinate functions that span the given manifold. For example, we cannot choose coordinates nicely over the surface of the earth: longitude, for instance, is not defined at the poles. This leads us to the concept of a chart. Given any manifold $M$ of dimension $N$ and a point $P$ in $M$ with a neighborhood $C$, a chart is a mapping (i.e., a function) that transforms $C$ into an $N$-dimensional Euclidean space $R^{n}$.

The coordinate functions of the chart are the real-valued functions on $C$. That is, they effectively become the values of the coordinate. Through the use of mapping associated with the chart, a "local" coordinate system is established. It is easy to see that such open neighborhoods must have overlaps, and that in those areas in which there is an overlap, the chart must satisfy a compatibility condition. All points on the manifolds are to be included in at least one chart. The collection of all admissible charts is called an atlas.

\section{TANGENT VECTOR, CONTRAVARIANT VECTOR}

A tangent vector has been defined as an $n$-tuple (i.e., $\left[T_{1}, T_{2}, T_{3}, \ldots\right]$ ) with a point of origin which transforms according to certain rules. However, more fundamental than the components of a vector is the vector itself. It is a geometric object with a meaning independent of all coordinates. Coordinates enter the picture when analysis is done on a computer. One view of tangent vectors is that the tangent vector $T$ is a description of the local behavior of a parametrized curve, or is the directional derivative operator with respect to a given parameter such as arc length $s$ defined on a given curve:

$$
T=d / d s
$$

This defines the tangent vector as a rate of change. However, this rate of change will not be defined absolutely, because there is no natural sense of distance on a manifold. The speed (i.e., the absolute magnitude of the rate of change) will be relative to other curves having the same direction, unless a metric (i.e., a method of measuring distance) is defined on the manifold of interest.

COTANGENT VECTOR, COVARIANT VECTOR, 1-FORM

A cotangent vector is a linear, real-valued function of a tangent vector. This means the following: a cotangent vector at a point $p$ on a one-dimensional manifold $M$ associated with a vector $V$ at $p$ is a real number, which we call $W(V)$. This notation expresses the idea that $W$ is a function of 
vectors. Cotangent vectors satisfy the axioms of a vector space and are dual to the tangent vectors. Therefore, it is possible to regard the tangent vectors as functions of cotangent vectors and vice versa. The tangent vector and cotangent vector can be related to each other through the use of a metric. A common cotangent vector or 1-form is the angular velocity of a rigid body. A second example is the gradient of a function (a scalar-valued vector field).

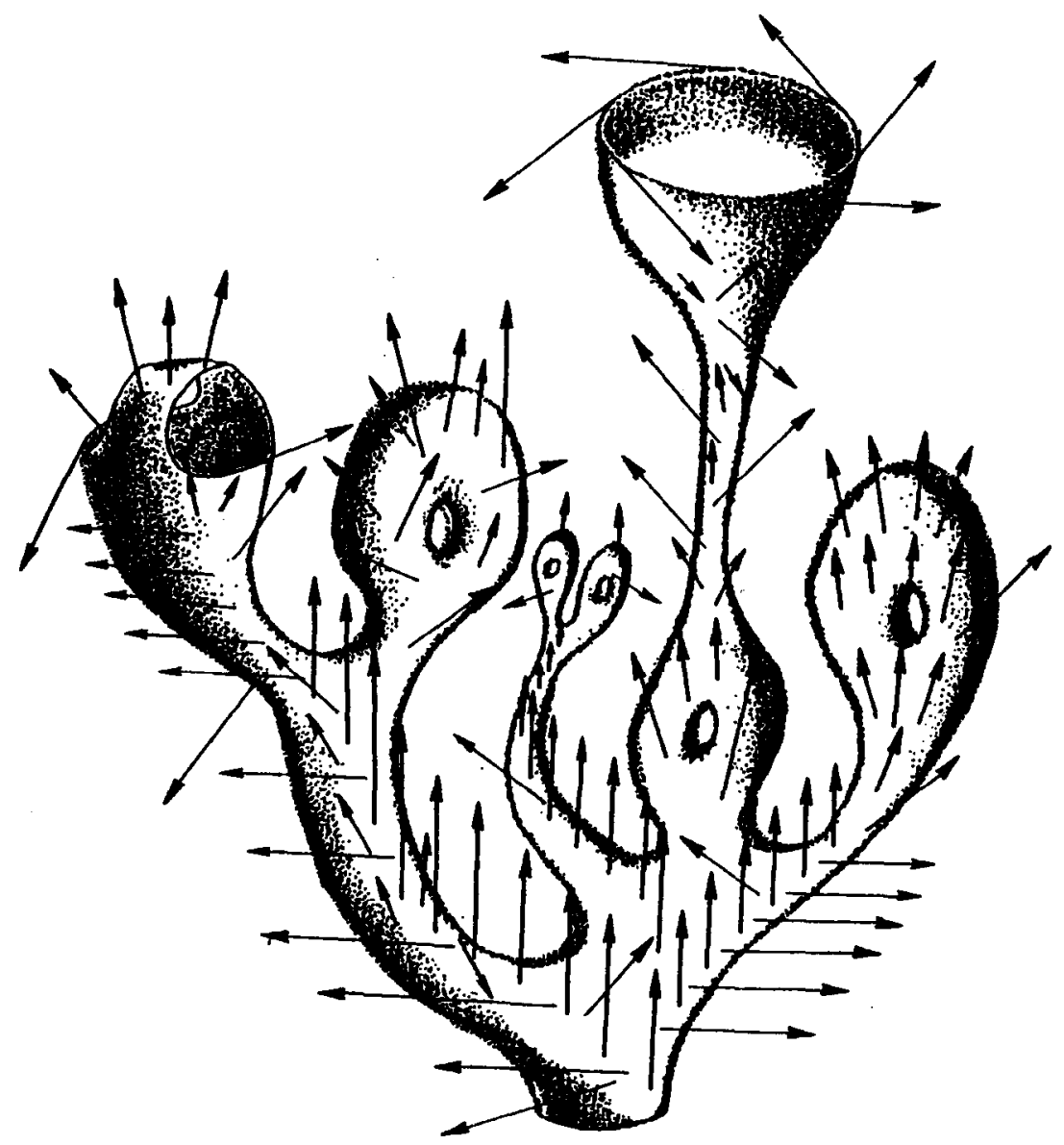

Fig. 1. A five-dimensional fiber bundle, composed of the base manifold, represented by the amorphous two-dimensional surface illustrated by the plant-like structure. The field variables are represented by the vectors which have their points attached to the two-dimensional surface. It is clear that all possible vectors cannot be represented in the figure: the price we pay for living in a three-dimensional world. Therefore, just a few of these vectors are represented. 
Although many treatments of vector calculus call the gradient a vector, it is more proper to label it a covector, that is, the gradient of a function as one moves in the tangent direction. Therefore, the gradient of a function is a function of two vectors: the function itself and the tangent vector.

\section{FIBER BUNDLES}

The field is a common mathematical structure used extensively in physics and mechanics. A field consists of attaching to each point, or a set of points, in a given manifold some geometric object, such as a tangent or cotangent vector. In general, the field can be viewed as a simple extension of a function at each given point. With this view, any function is a hypersurface in the larger space of the manifold, consisting of the base space (i.e., the manifold in which the points are defined) and the function. We now have a new

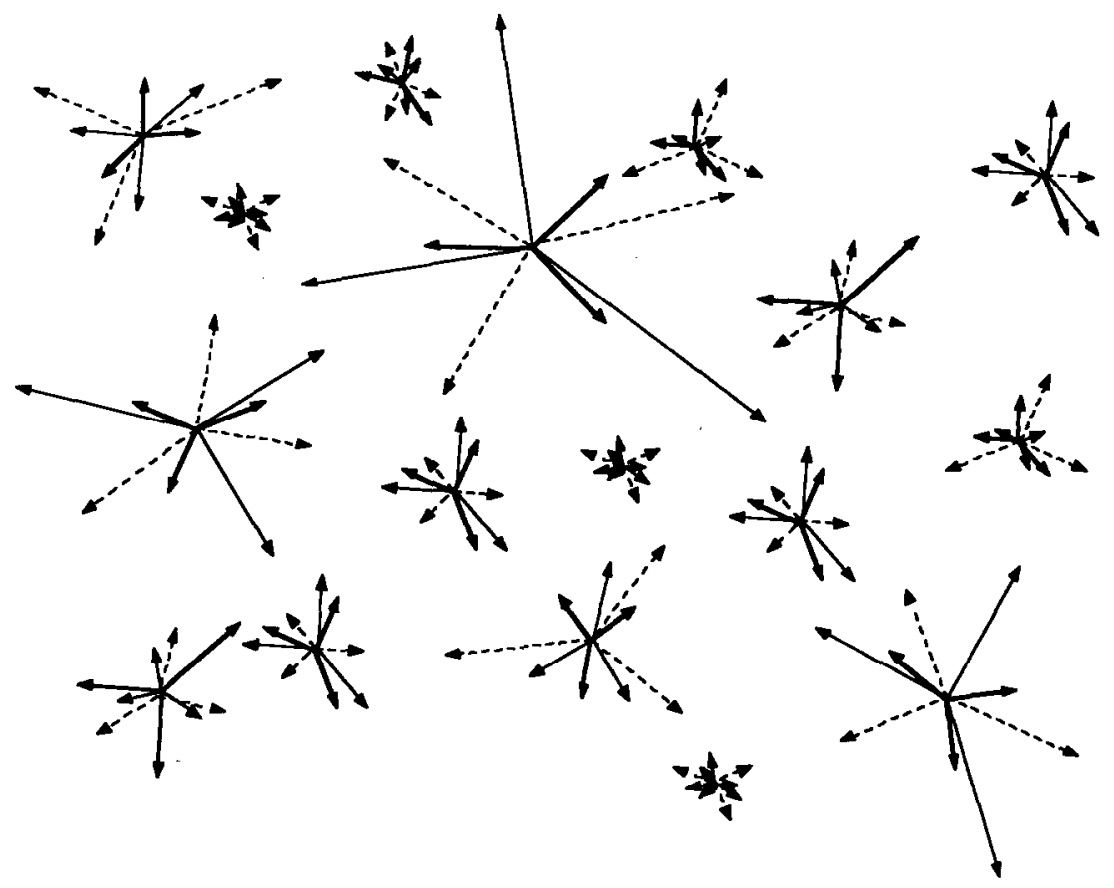

FIG. 2. A schematic representation of a section of the frame-bundle manifold. Each point in the base space has all possible orthogonal frames attached to it. We have sacrificed precision by displaying only a few of the points in the base manifold with a few frames at each point. This is obviously a problem when attempting to describe a 12-dimensional structure (three-dimensional base space, nine-dimensional attached field variables) using a two-dimensional surface. 
manifold composed of the original manifold (i.e., the base space) and the field variables which form independent coordinates (i.e., degrees of freedom) (Figure 1). This manifold with the increased dimensions is an example of a fiber bundle. A fiber bundle is a pair of manifolds $F$ and $B$ and a mapping *. The space $B$ is the base space, the manifold $F$ is the space in which the function is defined, and * is the mapping that assigns to each point in $F$ the point in $B$ where that field variable is defined. For any point $p$ that is an element of $B$, the manifold ${ }^{*-1}(p)$ is called the fiber over $p$. Three fiber bundles of interest are the cotangent bundle, the tangent bundle, and the frame bundle (Figure 2).

\section{MOVING FRAME}

A manifold can be studied by the use of the frame bundle, also known as the principal bundle. At each point in a given manifold, all frames (that is, a set of ordered bases for the manifold) are specified. The moving frame is a
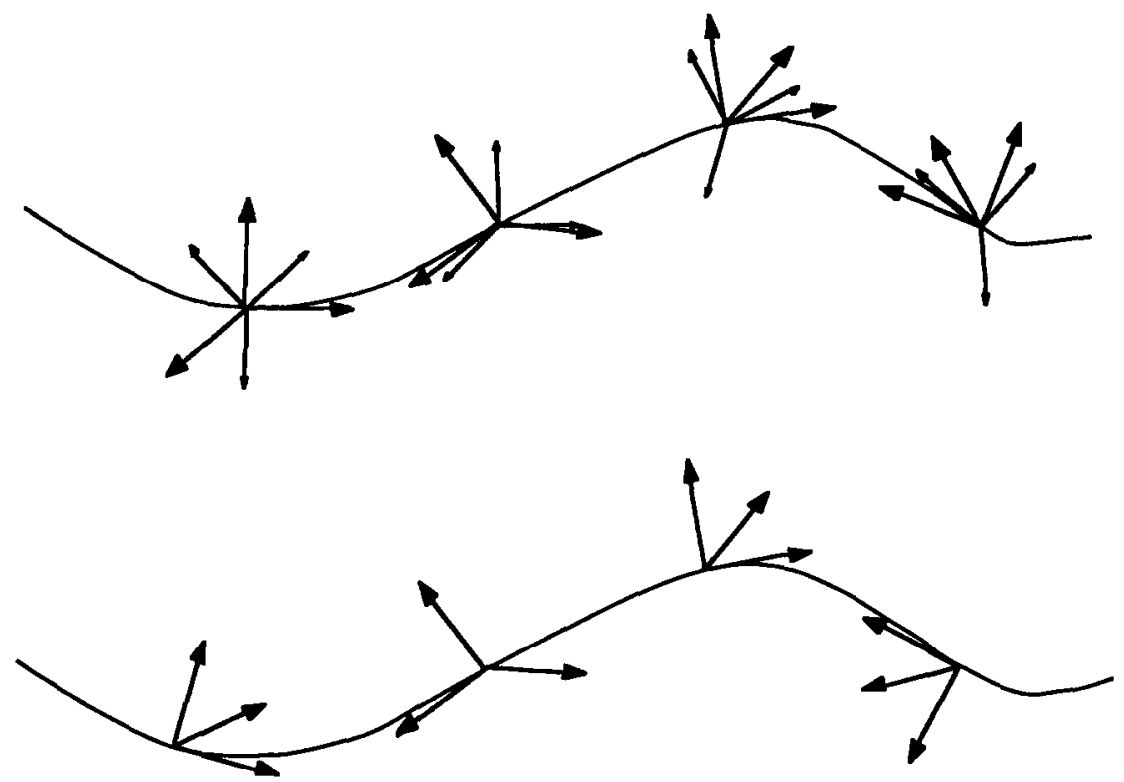

FIG. 3. Top: Schematic diagram of a subsection of the frame bundle. In this case, the base space is the curve and the field variables are all possible orthogonal frames on the curve. The moving frame is illustrated below, where only one frame is chosen at each point. Opposite: A core through the frame bundle showing some of the moving frames' subsections. This illustration is greatly simplified because it only shows a limited number of curves and moving frames. Representing the frame bundle schematically can be messy, since the various frames intersect one another and the curves of the moving frames haphazardly. 


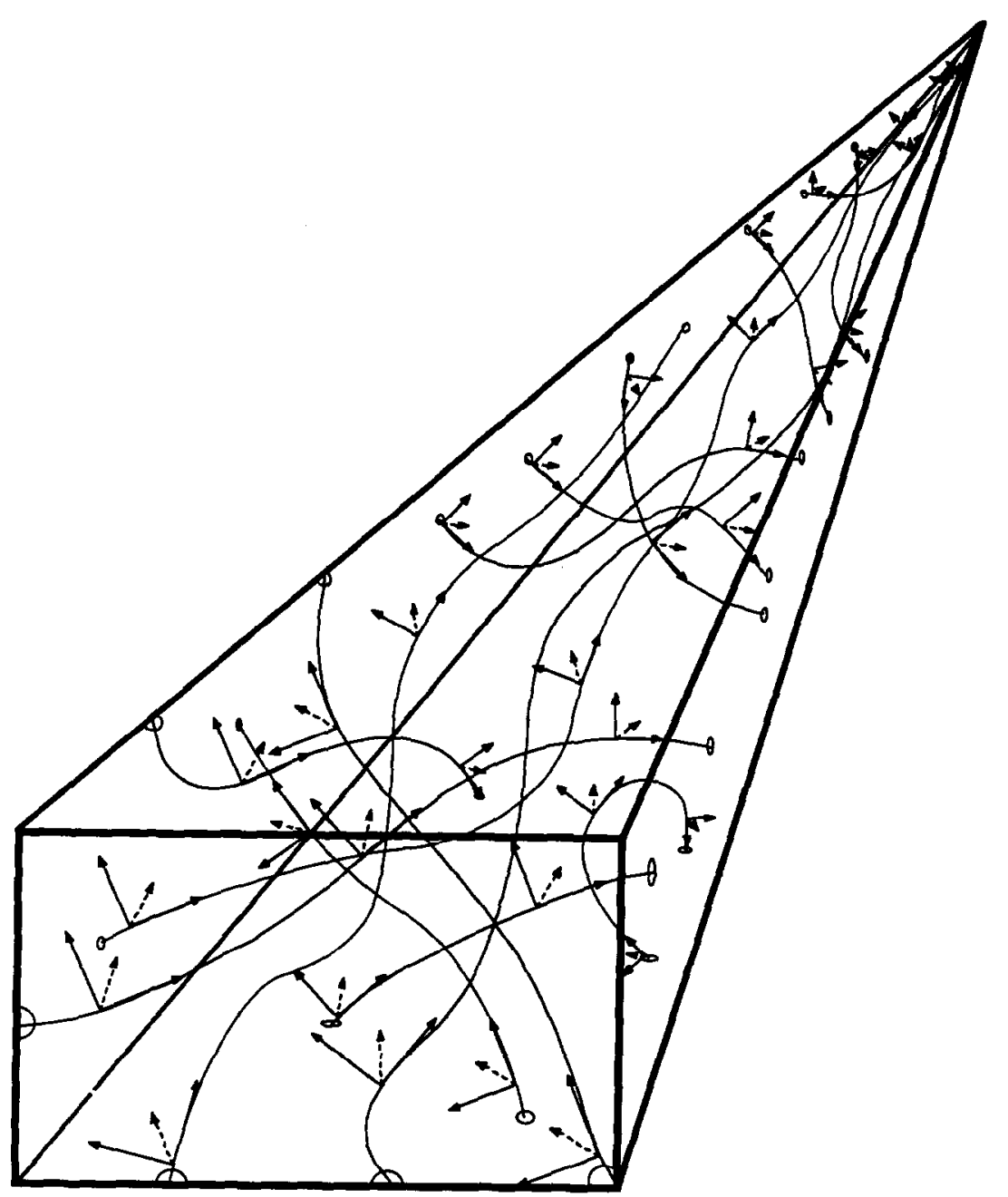

FIG. 3. Continued.

subsection of this manifold. An example of a moving frame is a one dimensional submanifold (i.e., a curve) embedded in Euclidean three-space, with a single orthogonal frame at each point on the curve (Figure 3 ). The base space of the principal bundle is Euclidean three-space with a fixed origin, so that points can be compared with one another. Assume that this curve is the path traced by the center of gravity of an arbitrarily rotating rigid body as it moves through space (Figurc 4). The subsection of the frame 


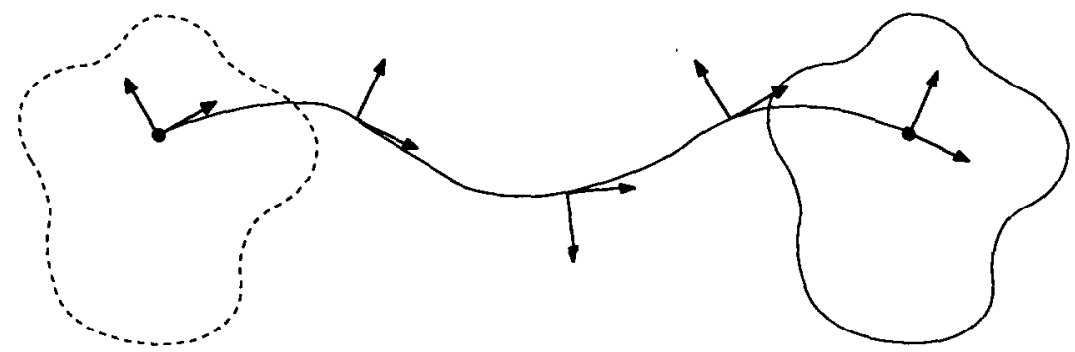

FIG. 4. A curve (one-dimensional manifold) traced in space as time progresses and the material body moves forward.

bundle will then consist of the frames attached to the curve and fixed within the rigid body. The rate of change of the moving frames as the curve is traveled can then be used to characterize the rotation of the rigid body (e.g., angular velocity is a cotangent vector associated with the rotating frame). Therefore, the submanifold of interest is a continuous function of position is three-space with a given frame at each point. However, the moving frames can be used in another way if an appropriate atlas has been defined on the manifold. The moving frame can then be used to characterize the manifold in question without reference to any embedding. In E. Cartan's theory [17], the basic idea is to express everything in terms of an arbitrary moving frame. The trick is to choose the moving frame most suitable to the particular problem at hand.

\section{HEAD IMPACT RESPONSE}

People are at risk for injury in any situation in which a head impact can occur. Because field accident information does not provide the level of detail necessary to ascertain mechanisms of injury, bioengineers use trauma experiments with human surrogates (i.e., cadavers or anesthetized animals) to document kinematic parameters so that mechanisms of injury can be better simulated, modeled, and verified, and ultimately so that people may be protected. In general, comparison of mechanical response between subjects is achieved by referring results to a standard, or set of standard reference frames.

\section{HEAD IMPACT TECHNIQUE}

Impacts to a given human surrogate are accomplished through the usc of an instrumented free-traveling mass. The type of impact delivered is con- 
trolled by adjusting the impactor mass, velocity, contact surface, and surface padding. Commonly, the impact mass of the striking piston is between 25 and $65 \mathrm{~kg}$. The velocity ranges between 1 and $10 \mathrm{~m} / \mathrm{s}$, the contact area between 20 and $100 \mathrm{~cm}^{2}$, and the padding between 0 and $10 \mathrm{~cm}$ in various forms.

During UMTRI head impact experiments, the surrogate head is instrumented with a nine-accelerometer array to measure three-dimensional motion (both linear and angular acceleration). For cadaver subjects, a triangular plate is secured by screws and acrylic plastic to the skull (Figure 5). Three triaxial accelerometer clusters are placed on the plate mount. The center of each triaxial accelerometer location and the center of the plate are marked by built-in lead beads, which form radiopaque targets that define the instrumentation frame field.

To determine the instrumentation frame's exact location and orientation in relation to the anatomical frame, a three-dimensional $\mathrm{x}$-ray technique was developed which requires taking two orthogonal radiographs of the instrumented head. The procedure requires the identification of four anatomical landmarks, which define the anatomical frame field, with four distinguishable lead pellets, plus the identification of four lead pellets inlaid in the plate (Figure 6). The anatomical landmarks include the two superior edges of the auditory meati and two infraorbital notches. The anatomical center is defined as the midpoint on the line connecting the two auditory targets. A computer program translates the instrumentation frame's center to that of the anatomical center.

Mechanical response and its relationship to mechanisms of injury is the key issue in trauma biomechanics. The biomechanics literature concerning mechanisms of injury to the head is a rich one [14]. However, despite conscientious speculation as to the mechanisms which produce head trauma and experimental and theoretical scrutiny of various hypotheses about it, considerable controversy still exists. Almost exclusively, the methods currently in use for describing the impact response of the head of human surrogates are based on paradigms from classical mechanical rigid body dynamics. It is common to assume that the head is a rigid body and to express the motion of the head in either the laboratory or anatomical (head) coordinate system. This reliance on classical rigid-body dynamics has restricted mechanical descriptors of head impact response to resultant angular and linear acceleration and velocity. For this reason the insight gained into head impact response has been limited. However, it is possible to use other paradigms from geometric theory to gain new insights into impact response. One method of doing this might be to view the impact response of the human body and its surrogates as geometric objects (i.e., as vectors, 1 -forms, tensors, manifolds) derived from experimental measurements. 

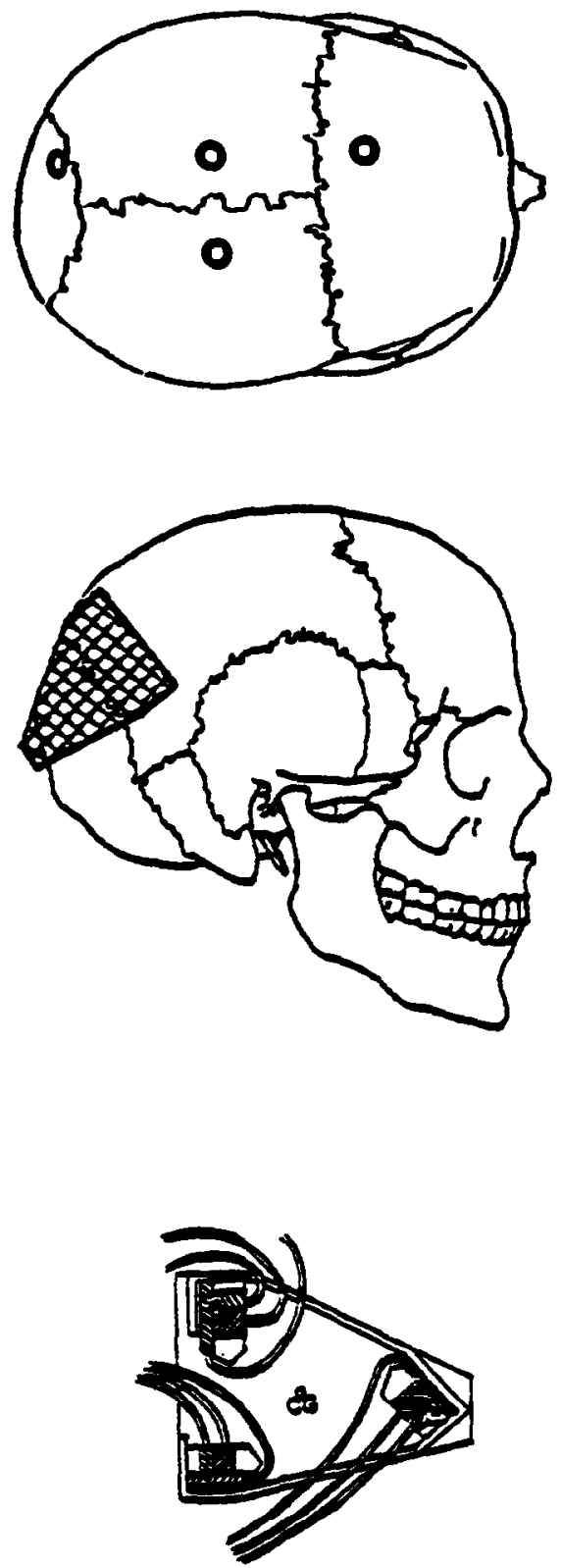

FIG. 5. The location of the UMTRI nine-accelerometer plates. The top image represents the top of the skull. The holes are locations of the subdural pressure transducers. The middle section shows the location of the plate on the skull for frontal impact. The lower image is a close-up of the nine-accelerometer plate with three triaxial accelerometers. 


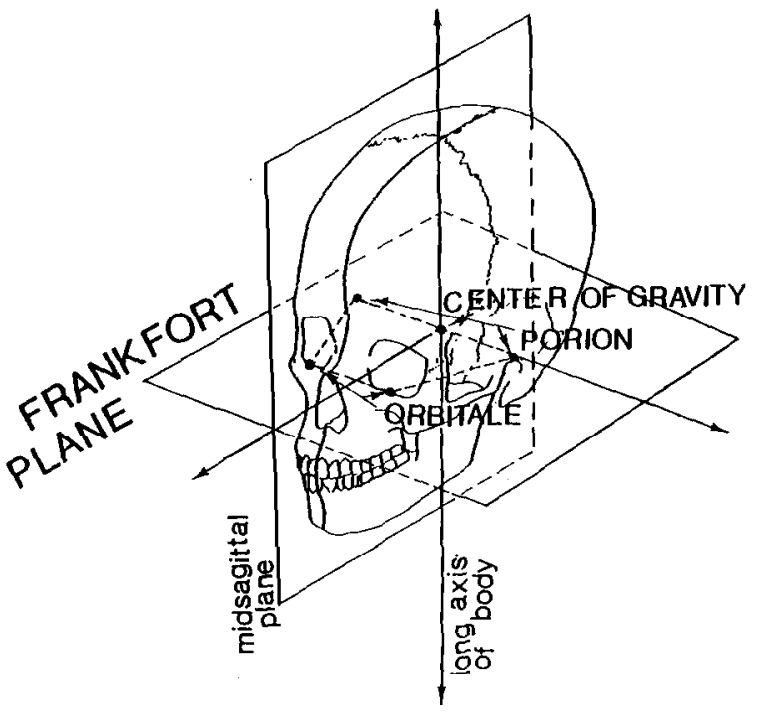

FIG. 6. An image of a skull and the anatomical landmarks used to define the anatomical center.

\section{MOVING FRAME OF THE HEAD}

As a material body moves in space, any point on it generates a path $C_{i}(s)$ (i.e., a curve, a one-dimensional manifold) in space (see Figure 7 where $C_{i}$ is a given curve and $s$ is the arc length). In head-injury research, we are interested in the description of the curves generated by the head as it moves. One point in particular has been chosen as the most important: the anatomi-

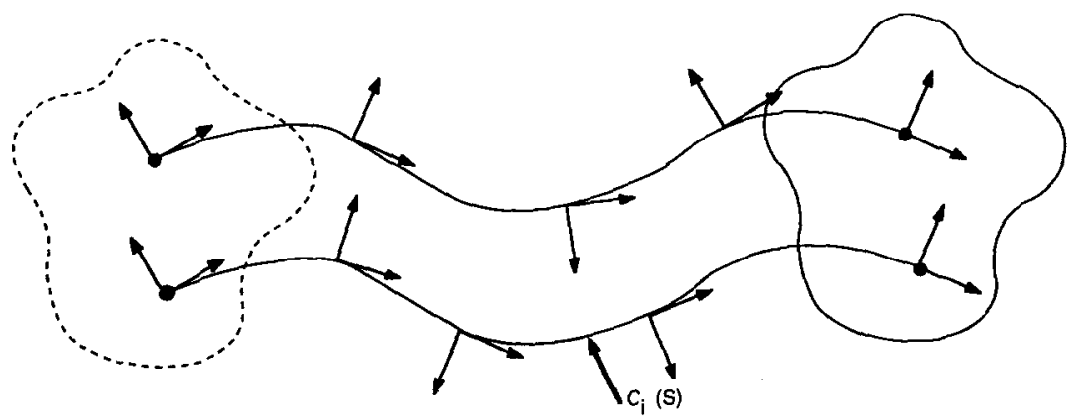

FIG. 7. A material body and the curve that is generated by the motion of the center of gravity as the body moves through space. 
cal center. Although too much emphasis has been placed on this point, it is convenient to use the path generated by it to form a base manifold for a subsection of the frame and tangent bundles.

One method of conceptionalizing the problem of head impact response is to view each point on the curve traced by the anatomical center during impact as the result of events on a continuous manifold. The continuous manifold of interest is the curve generated by the anatomical center $C_{\mathrm{ac}}(s)$ as it moves through space. The events on the continuous manifold of interest are the positions of the curve, defined in such a way that they pass from one event to another in a well-determined way. The idea of a curve as a collection of events was originated by Einstein, who said "to describe the time-history of an observer, describe what happens at each instant for that observer." The events through which an observer has passed form a simply extended manifold, having the essential characteristic that from any event in it, a continuous movement is possible in only two directions: forward and backward. To study more than one set of events, e.g., both position and velocity, one can now imagine that this manifold passes to another, completely different manifold (i.e., set of events) in a well-determined way, and then the events can form a doubly extended manifold. In a similar way, to include another event such as acceleration, one obtains a triply extended manifold when one imagines that a doubly extended manifold passes in a well-determined way to a completely different manifold. Therefore, in order to study the position, acceleration, and velocity of a moving point on the head, one can construct the necessary manifold or subsection of a fiber bundle.

The doubly extended manifold of the motion of the anatomical center of the head can be studied by use of a subsection of the frame bundle. The technique was originally proposed by Frenet in 1874 and by Serret in 1851 to understand the turning and twisting of curves in three-space $[5,7,18]$. To do so, it is instructive to look at manifolds in Euclidean three-space that are parametrized with arc length $S(t)$. One such manifold is a subsection of the tangent bundle derived from the nine-accelerometer array that includes the curve generated by the motion of the anatomical center and the tangent vector $d / d s$, consisting of unit vectors at each point on the curve (Figure 8). If one then takes the derivative ( $d / d s$, denoted as ') of this tangent vector $T$ of unit length, then this will measure the rate at which $T$ was changing. Since $T$ is of constant length, then its derivative is a measure of the way the curve is turning. Differentiation of $T \cdot T=1$ gives $2 T^{\prime} \cdot T=0$. Therefore, $T^{\prime}$ is always orthogonal to $T$. The plane that contains both the tangent vector $T$ and its derivative $k N$ is the oscillating plane. Here $k$ is the magnitude of the curvature cotangent vector field and gives a numerical measurement of the turning of the curve in Euclidean three-space $E^{3}$. A third unit vector, perpendicular to both $T$ and $N$, formed as the cross product $T \times N=B$, is 


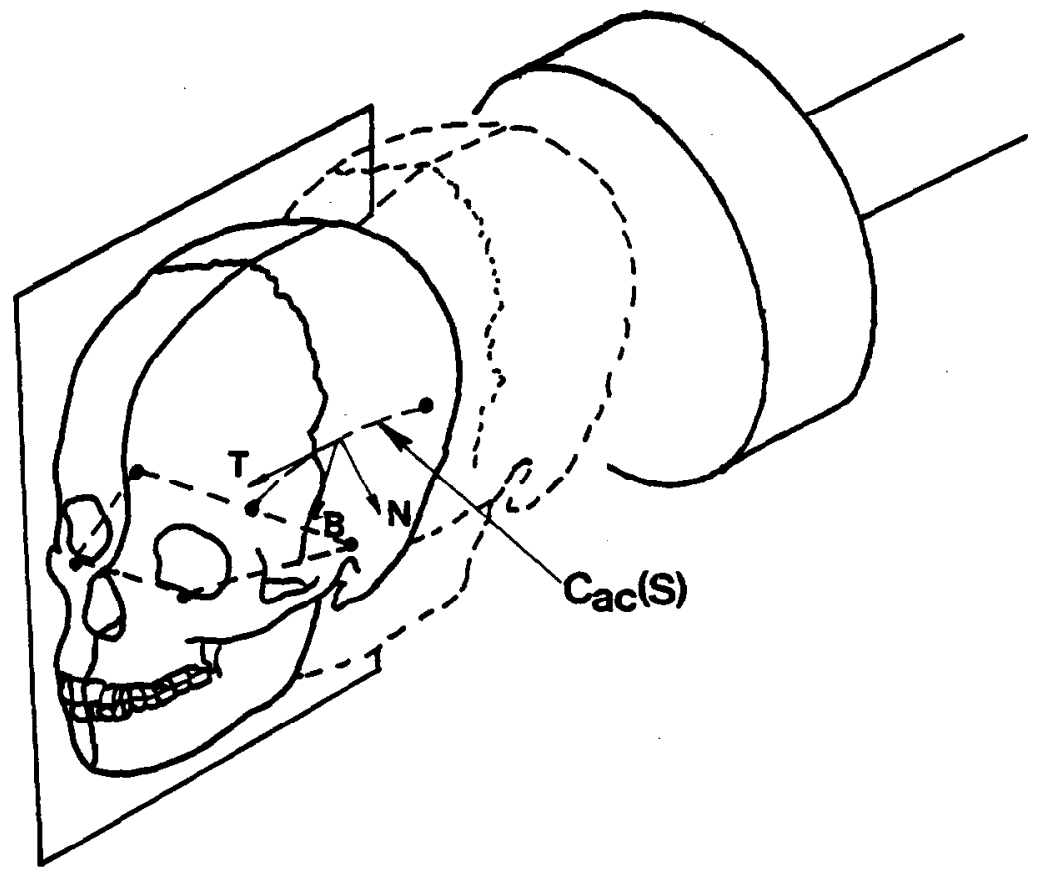

FIG. 8. The motion of the head with the tangent vector attached at the curve. The normal and binormal directions are also given. The subject has been impacted in the back of the head with the head bowing deep into the chest.

known as the binormal. It is important to understand how this frame changes with arc length as we move from one event to another. Since $B \cdot B=1$ and $B^{\prime} \cdot B=0, B^{\prime}$ must be a linear combination of $T$ and $N$. But $(B \cdot T)^{\prime}=0=$ $B^{\prime} \cdot T-B \cdot T^{\prime}$. Therefore, $B^{\prime} \cdot T=B \cdot T^{\prime}=B \cdot k N=0$, where $B$ must be a scaler multiple of $N$. We can now define a new function $t$, called the torsion, as

$$
B^{\prime}=-\tau N,
$$

where $\tau$ measure the rate of change of the oscillating plane (the plane containing the normal and tangential vectors). Of course, $\tau$ can only be defined where the curve has curvature. Thus, $\tau$ can be thought of as a measure of the rate at which the curve deviates from a plane curve and, like $k$, is also a cotagent vector. It is a simple matter to adapt these results to any form of reparametrization.

One of the most common parameters for a moving point is that of time $(d / d t$, denoted as $\cdot)$. The first and second derivatives of the curve with respect to time yield the velocity and acceleration, respectively. The magni- 
tude of the velocity vector is known as the speed, $v=d s(t) / d t$. The velocity vector then is

$$
\begin{aligned}
V & =\frac{d C_{\mathrm{ac}}}{d t} \\
& =\frac{d C_{\mathrm{ac}}}{d s} \cdot \frac{d s(t)}{d(t)} \\
& =v T .
\end{aligned}
$$

The acceleration vector contains components in both tangent $T$ and normal $N$ directions, with no acceleration in the binormal direction. Since velocity $=v \cdot T$, then the acceleration is

$$
\begin{aligned}
A & =\dot{V} \\
& =\dot{v} T+v \dot{T} \\
& =\dot{v} T+v^{2} k N .
\end{aligned}
$$

Thus, the tangent components measure the rate of change of speed of the curve, while the normal component measures the rate of change of direction of the velocity vector. $k$ measures how sharply the curve changes in the tangent direction. The derivative of the moving frame with respect to time becomes

$$
\left[\begin{array}{c}
T \\
N \\
B
\end{array}\right]=\left[\begin{array}{ccc}
0 & k v N & 0 \\
k v T & 0 & \tau v B \\
0 & \tau v N & 0
\end{array}\right],
$$

here $k v$ and $\tau v$ are the angular velocities of the moving frame.

In principal every geometric problem involving motion along a curved path can be solved by means of the Frenet-Serret method. For simple cases, it may be possible to record the acceleration data and express them in a conventional form. Examples of such instances are: for zero curvature, the motion of a point will be a straight line; for positive curvature and zero torsion, the motion will be in a plane; and for constant curvature with zero torsion, the motion will be in a circle (Figure 9). For more complicated motions, which are common even in simple head impact experiments, it is desirable to be able to classify the types of motion in convenient manner. The moving-frame approach provides such a manner.

It is important to keep in mind that the results obtained in head impact experiments rely on digitized transducer time-histories. Because of this, we never get the completely smooth manifold that is desired. Noise, both of 

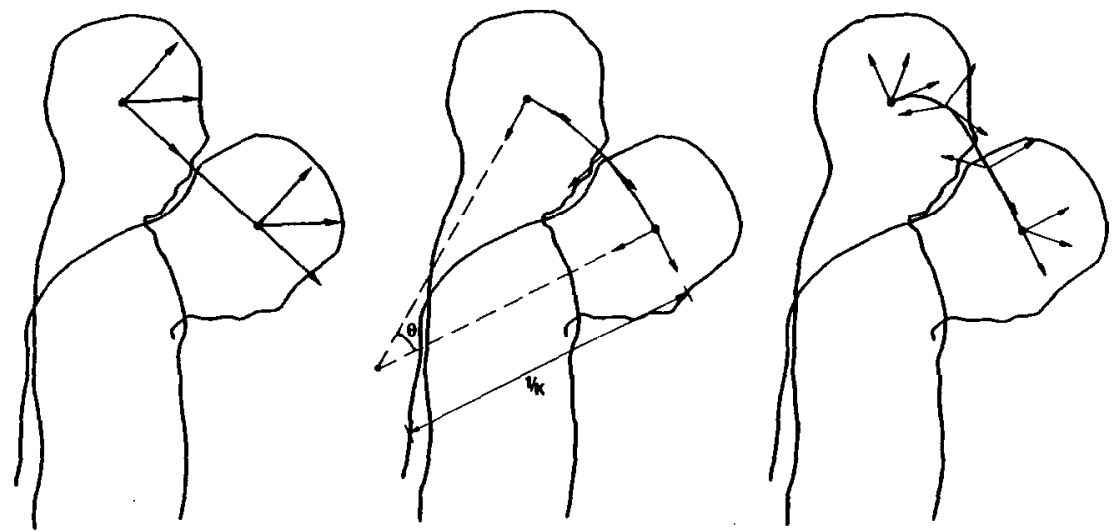

FIG. 9. Schematic representation of three impacts. The head is impacted from the back, forcing it forward. In the first image, the anatomical center moves in a straight line with zero curvature and zero torsion. In the second image, the head moves in a circular arc with constant curvature. The final image represents a more complicated motion with changing curvature and changing torsion.

high and low frequency, can enter into the data, leading to erroneous results. In addition, when dealing with digitized experimental results that are expressed as geometric objects such as vectors, manifolds, bundles, and subsections of frame bundles of moving frames, the data are obtained through numerical calculation executed by a computer which does not recognize the subtle aspects of the topology, but only the numbers entered into its working memory. This means that, in many cases, it may be conceptually desirable to develop and use the full power of geometric techniques. However, computational procedures may hinder the approach. One method of calculating the curvature and torsion follows:

$$
\begin{aligned}
& \kappa=\frac{\dot{C}_{\mathrm{ac}}(t) \times \ddot{C}_{a c}(t)}{\left|\dot{C}_{\mathrm{ac}}(t)\right|^{3}}, \\
& \tau=\frac{\left[\dot{C}_{\mathrm{ac}}(t) \times \ddot{C}_{\mathrm{ac}}(t)\right] \cdot \ddot{C}_{\mathrm{ac}}(t)}{\left|\dot{C}_{\mathrm{ac}}(t) \times \ddot{C}_{\mathrm{ac}}(t)\right|^{2}},
\end{aligned}
$$

where $C_{\mathrm{ac}}(t)$ is the curve, $\dot{C}_{\mathrm{ac}}(t)$ the velocity, $\ddot{C}_{\mathrm{ac}}(t)$ the acceleration, etc. The operation of integration to obtain velocity from acceleration is well defined, as are the cross and dot procedures. If the signal-to-noise ratio is not high 
$-\mid \begin{aligned} & \text { IMPACT } \\ & \text { FORCE } \\ & (\mathrm{N})\end{aligned}$

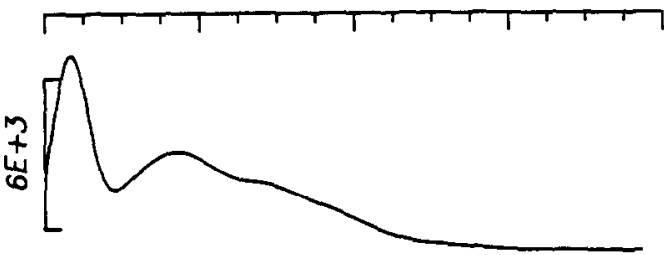

$\sim\left\{\begin{array}{l}\operatorname{LN} A C C \\ \operatorname{TAN}(T) \\ (\mathrm{m} / \mathrm{s} / \mathrm{s})\end{array}\right.$

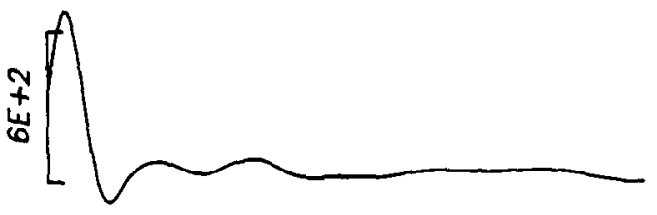

$m \mid \begin{aligned} & \operatorname{LIN~ACC} \\ & \operatorname{NOR}(\mathrm{N}) \\ & (\mathrm{m} / \mathrm{s} / \mathrm{s})\end{aligned}$

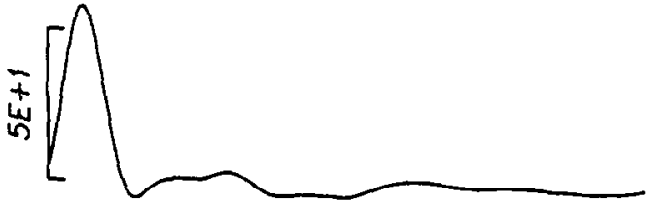

$\nabla \mid \begin{aligned} & \text { CURVTURE } \\ & \text { KAPPA } \\ & (1 / \mathrm{m})\end{aligned}$

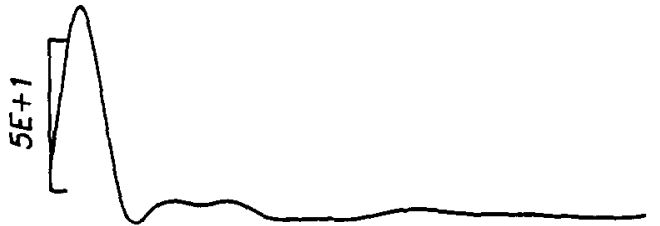

$n \mid \begin{aligned} & \text { TORSION } \\ & \text { TAU } \\ & (1 / \mathrm{m})\end{aligned}$

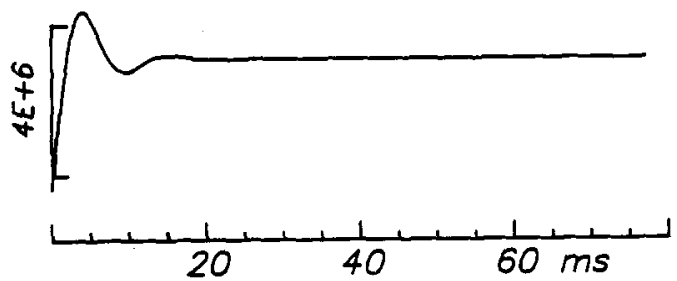

Fig. 10. A time history of an impact to the head. Variable 1 is the impact force. Variable 2 is the tangential acceleration. Variable 3 is the normal acceleration. Variable 4 is the curvature. Variable 5 is the torsion.

enough, the differentiation of the acceleration can lead to errors. However, in most cases this is not a problem.

When the data have been expressed in the moving frame of Frenet-Serret, the following has been observed:

1. The motion of the head during contact of the impactor with the head is three-dimensional (i.e., has significant curvature and torsion). (See Figures 10, 11.) 
$-\mid \begin{aligned} & \text { IMPACT } \\ & \text { FORCE } \\ & (\mathrm{N})\end{aligned}$

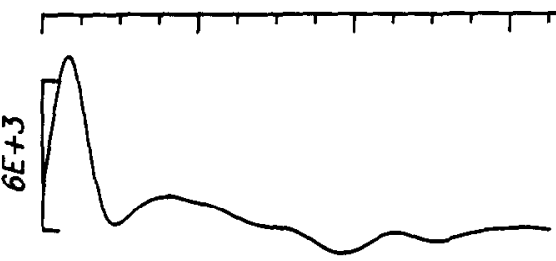

$\checkmark \mid \begin{aligned} & \operatorname{LIN~ACC} \\ & \operatorname{TAN}(T) \\ & (\mathrm{m} / \mathrm{s} / \mathrm{s})\end{aligned}$

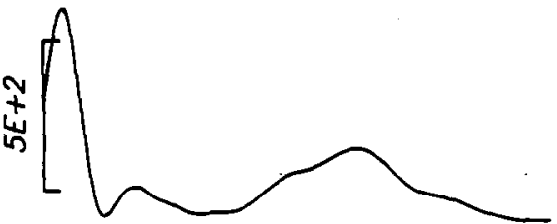

$m \mid \begin{aligned} & \operatorname{LIN~ACC} \\ & \operatorname{NOR}(N) \\ & (\mathrm{m} / \mathrm{s} / \mathrm{s})\end{aligned}$

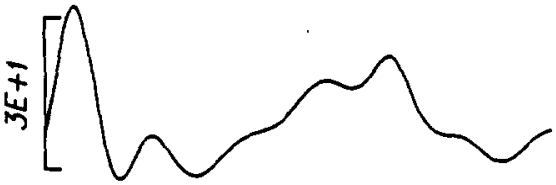

$+\mid \begin{aligned} & \text { CURVTURE } \\ & \text { KAPPA } \\ & (1 / \mathrm{m})\end{aligned}$

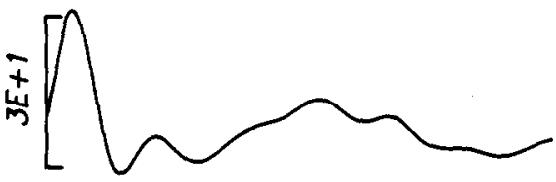

م $\left\{\begin{array}{l}\text { TORSION } \\ \text { TAU } \\ (1 / \mathrm{m})\end{array}\right.$

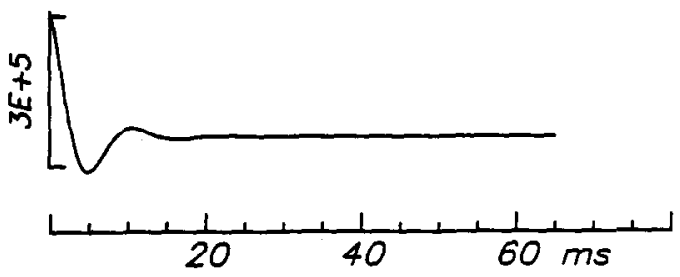

FIG. 11. A time-history for an impact to the head. Variable 1 is the impact force. Variable 2 is the tangential acceleration. Variable 3 is the normal acceleration. Variable 4 is the curvature. Variable 5 is the torsion.

2. The motion of the head after contact of the impactor with the head is primarily two-dimensional (i.e., has significant curvature with little torsion). (See Figures 10,11.)

3. Even though the motion of the head is three-dimensional during contact of the impactor with the head, the primary acceleration is in the tangential direction. Some aspects of this can be seen from Figures 10, 11.

This last point needs some elaboration. The tangential acceleration is the rate of change of speed of the velocity (i.e., the rate of the resulting velocity). 
This permits a comparison between tests. If one constructs a curve in a six-dimensional space (the tangent bundle), one can then use that curve and vectors attached to that curve (by extending the manifold) to make comparisons between impact tests. The implication here is that in an impact situation, the energy transferred to the head from the impactor manifests itself primarily as a change in speed as opposed to a change in direction. Therefore, even though the motion of the anatomical enter is three-dimensional in the Euclidean sense, the best one-dimensional estimation in the Riemannian sense is obtained through a one-dimensional curve embedded in the tangential bundle. This conclusion has led to the defining of a second subsection of the principal bundle, which I have called the principal-direction moving frame.

With nine accelerometers it is possible to describe a chart in Euclidean three-space, and thus construct the Frenet-Serret frame for a head impact. However, with a triaxial accelerometer cluster that is arbitrarily located on the head, this cannot be done. An approximation of the Frenet-Serret frame can be obtained by finding the direction of acceleration in the reference frame of the moving triaxial accelerometer cluster which contains most of the acceleration, plus a second direction which contains the next most acceleration. These directions can then be used as an approximation of the tangential and normal directions, respectively, permitting comparison between subjects with triaxial accelerometer clusters.

A further use of this relationship depends on the fact that the tangential acceleration and, to a reasonable approximation, the principal-direction acceleration are the change in the resulting velocity. This information can then be used to generate comparisons in the frequency domain through the use of mechanical impedance.

\section{MECHANICAL IMPEDANCE}

With blunt impacts, the relationship between a function on a given curve and a function on another given curve in the fiber bundle can be expressed in the frequency domain through the use of a transfer function. A fast Fourier transformation of simultaneously monitored transducer time histories can be used to obtain the frequency response functions of the head impact force and the velocity along the curve of motion of the anatomical center. Once obtained, the transfer function of the form

$$
(Z)(i \omega)=\frac{\mathbf{F}[F(t)]}{\mathbf{F}[V(t)]}
$$

can be calculated from the transformed quantities, where $\omega$ is the given frequency and $F[F(t)]$ and $F[V(t)]$ are Fourier transforms of the impact 
force and velocity along the curve. This particular transfer function is closely related to a mechanical transfer impedance [19], which can be defined as the ratio between the harmonic driving force and the corresponding velocity of the point of interest. Mechanical transfer impedance is a complex-valued function which can be described simply by its magnitude and phase angle. Once obtained, this transfer function can be used to establish restriction (i.e., an equation defined on the curve) to help characterize head impact response. Restriction on the manifold of interest adds structure to that manifold.
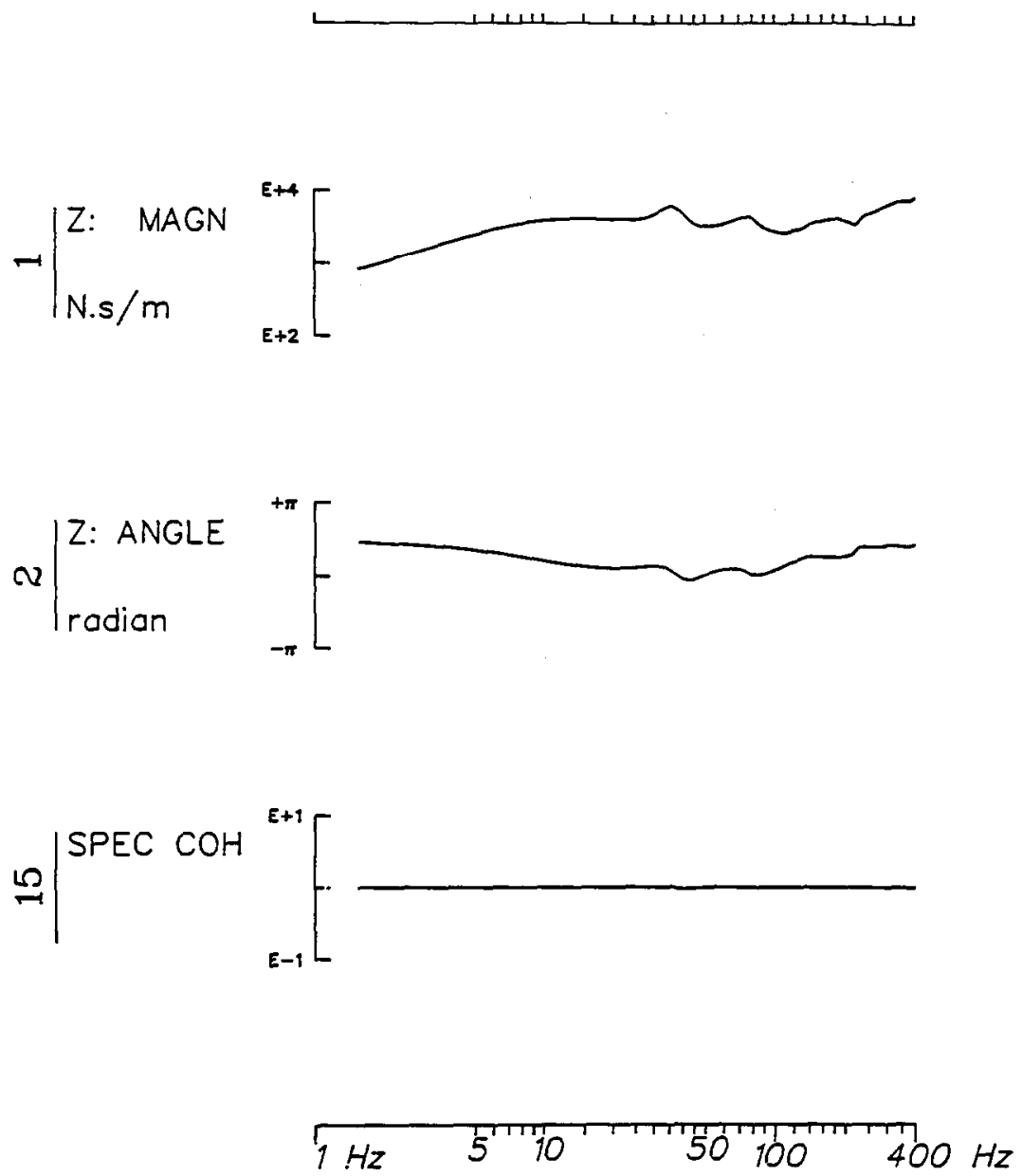

FIG. 12. A mechanical-impedance transfer function generated from force divided by tangential acceleration: first example. 

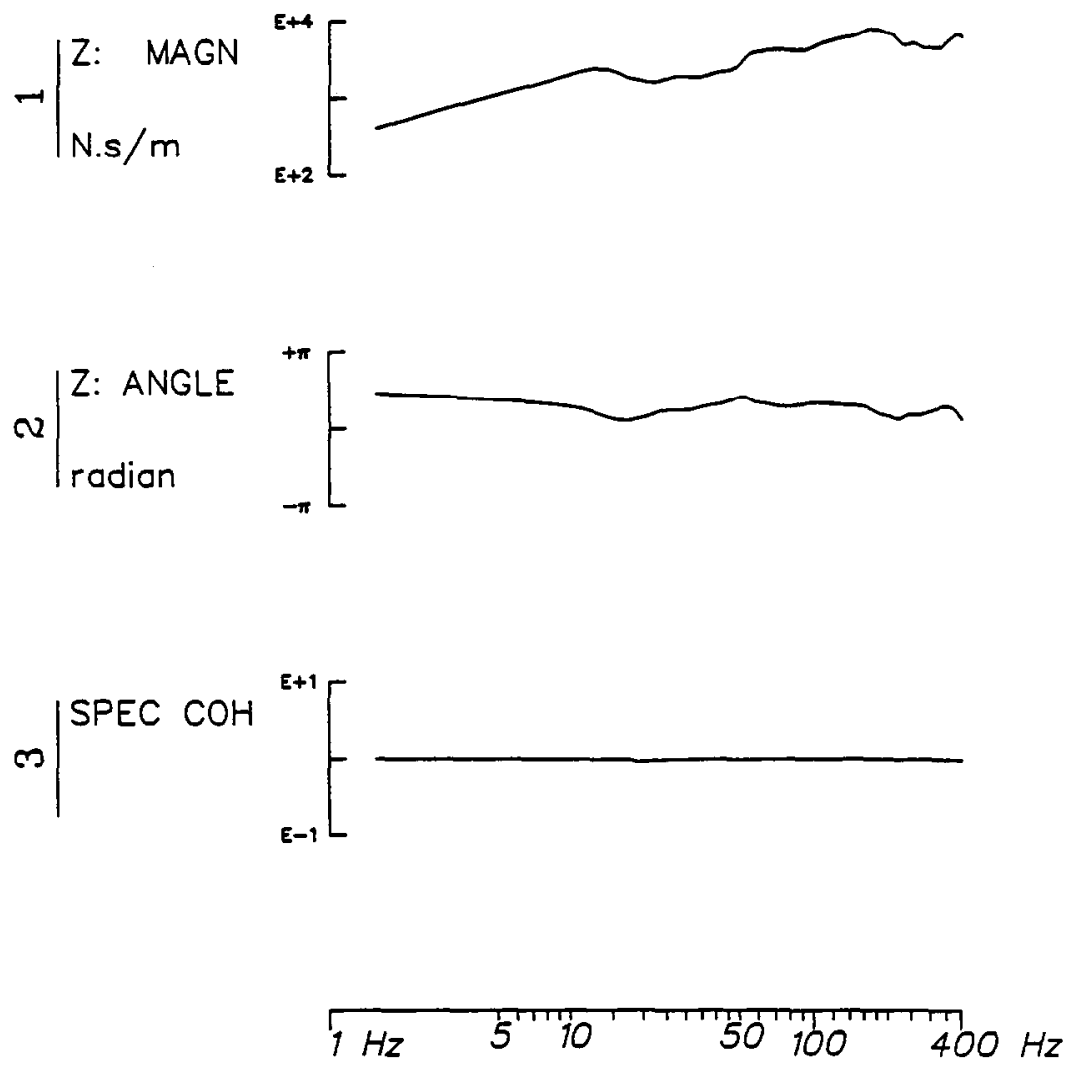

FIG. 13. As in Figure 12: second example.

Embedding structures in a manifold is a way of defining the physics of the space of interest or (analogously to classical mechanics) generating a model of the physical system. The inverse method is a common method to generate a model or to understand the physics at hand from the experimental data (see [20-23]). In a similar matter, an equation or model defined on the curve that adds structure to the subsection of the tangent bundle of interest can be generated. Figures 12 and 13 are the mechanical impedances for the force divided by velocity along the curve of the anatomical center of a human cadaver during head impact. 


\section{CONCLUSIONS}

The qualitative understanding of the physical world can depend on which paradigms are used to express first principles as well as which first principles are used. The geometric method offers a different approach as well as a different framework to address classical and experimental mechanics. Although at first the paradigms and techniques may seem foreign to those working in experimental mechanics, they can offer insights into the mechanics under study that may not occur using the classical approach. One such example is head impact response.

In biomechanics experiments, such as head impact experiments, it is virtually impossible to align accelerometers and load cells in the same way for all test subjects. This problem is eliminated by using geometric techniques. The accelerometer which is mounted on the cadaver can be aligned in an arbitrary manner (providing that there are at least three transducers which are mutually orthogonal). Comparisons between subjects can be made using frame independent qualities such as tangential or principal acceleration. This will then eliminate a lot of the experimental error associated with the initial orientation of the transducers.

The author wishes to extend his gratitude to Nabih Alem for the implementation of the basic software and to Robert Bennett and Donald Huelke for their feedback. The author is also grateful to Patricia Kaiker, Richard Lehman, Valerie Karime, Steven Richter, Lillian Kim, Wendy Gould, and Colleen Jones without whom this manuscript could not have been completed. A special "thank you" is due to Jeffrey Marcus for his critical review of the manuscript.

The research in this paper was partially funded by the Department of Transportation, National Highway Traffic Safety Administration, Contract Nos. DTNH22-83-C-07095 and DTNH22-83-C-1701

\section{REFERENCES}

1 R. Abraham, and J. E. Marsden, Foundations of Mechanics, Benjamin/Cummings, Reading, Mass., 1978.

2 R. L. Bishop and S. I. Goldberg, Tensor Analysis on Manifolds, Dover, New York, 1980.

3 H. Goldstein, Classical Mechanics, Addison-Wesley, Reading, Mass., 1980.

4 C. W. Misner, K. S. Thorne, and J. A. Wheeler, Gravitation, San Francisco, 1973.

5 B. O'Neill, Elementary Differential Geometry, Academic, New York, 1967.

6 G. Sorani, An introduction to Real and Complex Manifolds, Gordon and Breach, New York, 1969.

7 M. Spivak, A Comprehensive Introduction to Differential Geometry, Vol. 2, Publish or Perish, Inc., Berkeley, Calif., 1979.

8 G. S. Nusholtz, et al., Comparison of epidural pressure in live anesthetized and postmortem primates, in 7th International Workshop on Human Subjects for Biome- 
chanical Research Proceedings, Coronado, Calif, Washington, D.C., distributed by the National Highway Traffic Safety Administration, 16 Oct. 1979, pp. 175-200.

9 G. S. Nusholtz, J. W. Melvin and N. M. Alem, Head impact response comparisons of human surrogates, in 23rd Stapp Car Crash Conference Proceedings, 1979, pp. 497-542.

10 G. S. Nusholtz, et al., Response of the cervical spine to superior-inferior head impact, in 25th Stapp Car Crash Conference Proceedings, 1981, pp. 197-240.

11 G. S. Nusholtz, N. M. Alem, J. W. Meivin, Impact response and injury of the pelvis, in 26th Stapp Car Crash Conference Proceedings, 1982, pp. 103-144.

12 G. S. Nusholtz, et al., Cervical spine injury mechanisms, in 27th Stapp Car Crash Conference Proceedings, 1983, pp. 179-188.

13 G. S. Nusholtz, J. W. Melvin, and P. Lux, The influence of impact energy and direction on thoracic response, in 27th Stapp Car Crash Conference Proceedings, 1983, pp. 69-94.

14 G. S. Nusholtz, et. al., Head impact response-skull deformation and angular accelerations, in 28th Stapp Car Crash Conference Proceedings, 1984, pp.41-74.

15 G. S. Nusholtz, et al., Thoracic response to frontal impact, 29th Stapp Car Crash Conference Proceedings, 1985, pp. 17-48.

16 G. S. Nusholtz, et al., Thoraco-Abdominal response to steering wheel impact, in 29 th Stapp Car Crash Conference Proceedings, 1985, pp. 221-267.

17 E. Cartan, Leçons sur la Géométrie des Espaces de Riemann, 2nd ed., Gauthier-Villars, Paris, 1946.

18 J. J. Stoker, Differential Geometry, Wiley-Interscience, New York, 1969.

19 C. M. Harris, and C. E. Crede, Shock and Vibration Handbook, McGraw-Hill, New York, 1976.

20 R. L. Parker, Understanding inverse theory, Ann. Rev. Earth Planetary Sci. 5:35-64 (1977).

21 P. C. Sabatier, Inverse problems-an introduction, Inverse Problems. 1:i-vi (1985).

22 J. B. Bednar, R. Redner, E. Robinson, and A. Weglein, Conference on Inverse Scattering and Application, SIAM, Philadelphia, 1983.

23 F. Santosa, Y. H. Pao, W. W. Symes, and C. Holland, (Eds.), Inverse Problems of Acoustic and Elastic Waves, SIAM, Philadelphia, 1984. 